\title{
Comparative Study of Safety Awareness Techniques
}

\author{
Mohamed Ismail. $\mathbf{S}^{1}$, Dr. Muthukumar. $\mathrm{K}^{2}$, Janardhan. $\mathrm{S}^{3}$ \\ ${ }^{1}$ PG Student, ${ }^{2}$ Professor, ${ }^{3}$ Assistant Professor \\ Department of Mechanical Engineering, \\ Bannari Amman Institute of Technology, Sathyamangalam, Tamil Nadu, India
}

\begin{abstract}
Employee safety should hold enormous significance for each organization. First and foremost, each worker has the privilege to work in a safe and healthy environment. No employee or their families ought to need to endure as a result of avoidable danger in the work environment. This paper tells about, how importance safety awareness, the worker should know the work place and occupational related hazard, how to engage people safely, how people doing job safely without any hazard. $80 \%$ of accident caused by un safe act, eliminating this kind of accident we can improve safety awareness among workers. Some of the different methodology for improving safety awareness is the methodology include proper Education and Training, Conducting safety programs, Complimenting safe employees or safety reward, Employ safety incentive plan, Live demonstrations, Timely posting of safety information, awareness of the people from danger by means of establishing safety sings and Promoting Safety committee. These are the ways to alert the people from danger. If we are following this methodology 100 percentage of possible result expecting form worker as well as improving workplace safety also will be achieved.
\end{abstract}

Keywords: safety awareness, work place hazard, alert people form danger, methodology, un safe act.

\section{INTRODUCTION}

A human injury or death changes lives perpetually for families, friends, communities, and colleagues as well. Human accident and enduring is can not be measurable. DECREASING INJURIES REDUCES COSTS TO YOUR BUSINESS: If a worker is harmed at work, it costs the organization in lost work hours, expanded protection rates, laborers' remuneration premiums and possible litigation. Efficiency is lost when different laborers need to stop work to manage the damage. "safety first" has to be compelled among them and have to create selfawareness amoung that will help them and have to create self-awareness among that will help them to make a leaning of "first safety then work". [1]Training as "arranged endeavors to encourage the learning of particular OHS [occupational safety and health] capabilities. Past straightforward endeavors to transmit information, our definition incorporates a scope of endeavors intended to connect with trainees with the objective of influencing inspiration, attitudes, and conduct to improve worker health and safety at work. [2]A poster campaign is to decide if danger awareness among workers can be upgraded. Consideration was likewise paid to the part of the poster campaign in enhancing safety. The campaign was led in the shipbuilding industry and was coordinated fundamentally to laborers who were presented to risk related with the use of scaffolds.[3]A the small group activity method at a General Motors office in Linden conducting An educational programs tending to these issues was utilized in a training of 30 employee who might move toward becoming trainers. At that point, with some further help from Leopold, these 30 worker- trainers directed another ten classes in which 350 employee were trained. The training was so famous and viable, as indicated by Leopold, that the company denied additionally discharge time for the individuals who had not been trained.[4]In this examination we looked to inspect three hypotheses with respect to sorts of training and levels of OHS awareness furthermore, strengthening to take an interest in injury prevention. As hypothesized we watched that active training (workshops and outside training classes) had more grounded relationship with awareness than passive methods of training (web based training and work book). In accordance with our second hypothesis, we watched weaker 
relationship with training and strengthening than we improved the situation awareness, with just a single critical relationship between a mix of passive and active training and higher strengthening, contrasted with not accepting any training.[5]It has some time been perceived that open communication and continuous interactions amongst workers and supervisors are imperative hierarchical attributes, which different organizations with low accident rates from those with high accident rates Research has likewise demonstrated that safety communication was fundamentally connected with safety behavior, for example, consistence, safety knowledge, safety investment, and achievement of safety programs. [6]Incentives are a proactive approach connected by administration to improve the safety related exhibitions of workers. The utilization of Incentives, which incorporate both money related and nonfinancial prizes, can be useful for urging worker to be engaged with safety programs. Cases of monetary Incentives are cash or prizes, though nonfinancial fortification can be accomplished by positive evaluation, acknowledgment, or positive input [7]Borman and Motowidlo (1993) proposed two noteworthy parts of execution: task execution and contextual execution. These two parts of execution can be utilized to separate safety behaviors in the workplace. To begin with, in light of meanings of task execution, we utilize the term safety consistence to portray the center safety exercises that should be completed by people to keep up workplace safety. Second, in light of meanings of contextual execution, we utilize the term safety interest to portray practices. [8]The connections between safety culture and safety execution and whether there is simulative impact for safety execution have dependably been the point for investigating. In any case, it has been affirmed that the connection between safety culture and safety execution is qualitative and quantitative both from hypothetical investigation and experimental study. [9]OHS training alludes to arranged endeavors to encourage the learning of OHS-particular capabilities. OHS training can likewise engagelaborers and supervisors to end up more active in rolling out improvements that upgrade worksite safety [10]

\section{METHODOLOGY USED}

The following methodologies were put into use on a trial basis in edible oil manufacturer company and the response from the workers and the management was noted.

\subsection{Safety induction training}

Safety Induction Standard course furnishes laborers and contractual workers with an essential learning of work place health and safety lawful duties and procedures. It gives a diagram of basic safety issues and an essential comprehension of how to apply safety and emergency methods.

Safety induction ought to dependably be given to employees, trainees, contractual workers (including organization laborers) and volunteers, including those on work understanding.

\subsection{Education and Training}

Education and training are vital apparatuses for advising worker and supervisors about workplace hazard and controls so they can work all the more safely and be more profitable. Another part of Education and Training, in any case, is to provide laborers and supervisor with a more prominent comprehension of safety and health program itself, so they can add to its improvement and execution.

\section{Education and Training gives supervisor, employees, managers with:}

$>$ Knowledge and abilities expected to do their work safety and abstain from making dangers that could put themselves or others in danger.

Awareness and comprehension of workplace hazard and how to recognize, report, and control them.

$>$ Specialized training, when their work includes exceptional hazard.

\subsection{Toolbox training}

safety experts to share and trade free safety point resource particularly Toolbox Topics, Toolbox Talks and other free safety resource. In case you're similar to such a large number of organizations nowadays, the day by day safety meeting has demonstrated extremely effective in helping workers about the significance to remember safety in their day by day undertakings.

Toolbox Talks" , "Safety Chats" , "Rear end Meetings" or whatever your industry calls them is a short safety speak or meeting about a particular subject toward the start beginning of the shift. These discussions should be possible in anvariety of ways yet are regularly a concise (2-5 minute) intuitive discourse meeting on something safety related. 
Toolbox Topics are utilized to cover an variety of short safety training subjects and to remind employees every prior day they go to work, the significance of being protected

\subsection{Conducting safety programs with safety related term}

Game shows and quizzes which joins with safety rule and schedules can be coordinated inside the work space.

The games and questions let go ought to be from safety rule and regulations.

At the point when such games are conducted, employee take in more and upgrade their insight about safety in the workplace. The individuals who are uninformed about safety are excessively arranged by this philosophy.

\subsection{Safety reward or Reward employees for safe} behavior

It is continually a genuine propensity and an eager way to compliment a safe worker with the goal that others likewise watch the same.

At the point when a is supplemented safe employee he endeavors to spare every year and is a boost for other workers also.

Complimenting works superior then safety meetings and observations. Also, subsequently complimenting and rewarding are a protected method to help up workers towards safety.

Rather then reward high production focuses on that encourage speedy work with a contemptuous shrug towards safety, reward those worker who have taken after the entirety of your safety rules and have given productive work reliably. By putting an accentuation on safety rather than efficiency

\subsection{Assign Safety Champion}

They are usually conducted to focused employee attention on specific accident problem and frequently associated with a particular slogan or theme to maintain interest and visibility.

They may use media such as posters, banners, video types, booklets and a variety of written and oral communications.
They are aimed at increasing awareness, conveying information and changing attitudes in an effort to produce behavior change.

The intended effect of safety champion is same as that of behavior modification and programmes that attempt to get employees, supervisors and managers to make safety an integral feature of proficient jobperformance.

\subsection{Safety information should be reach employee}

It is constantly a good plan to cause a different place where safety related data is usable.

At the point when your organization controls a website, a separate portal can be conferred in the intranet only for safety information which makes coming up such safety data easy for the employees when they need.

The information can incorporate proposals, procedural manuals, accident investigation's findings, MSDS sheets and furthermore training videos.

At the point when such information easy to choose and it helps the worker a considerable measure when running for safety related information.

\subsection{Employee safety incentive plan}

To take out or build up safety plan, safety incentive program can be discovered.

These programe are fun and effective approaches to train employees with respect to safety at work.

The programe set them up with some little insights in regards to safety and how to work safely in the system.

\subsection{Live demonstrations}

To get people associated with your message about safety try to show live showings among employees.

Make the employees to show so the worker present can bring up their mistakes and help them enhance their method for working and take after safety. By thusly potential harm being caused to their bodies can be stayed away from.

\subsection{Timely posting of safety information}

The authoritative team can post videos, pamphlets and email with respect to safety measures to their employees. 
Get the message dribble feed on occasion so the worker knows that safety at work put is significant.

Despite the fact that employees are happy with their work constrain, convenient posting of such messages through email or notices influences them to review a moment about safety. In this way workplace safety can be improved.

\subsection{Establishing safety signs}

Purpose of Signs: Safety signs give vital particular safety information from warning of danger, giving instruction and what kinds of personal protective equipment required for particular job.

Danger Signs: These signs caution of particular hazard and radiation hazard. OSHA necessitates that all Danger Signs be of a similar sort of outline. A danger sign ought to be obvious to all. employees must be told that danger signs show impending hazard and that unique precautionary measures are necessary.

Caution Signs: Caution signs are utilized just to caution against potential hazards or to alert against unsafe practices. All workers must be told that caution signs demonstrate a possible danger against which appropriate safeguard should be taken.

\subsection{Emotional stories work better}

To expand workplace safety, stories can be a smart thought as a human mind lean towards stories.

Emotional stories should emotionally touch heart and be more related to normal life, at same time expressing story have littlehumor to reach human brain and it help worker focus on the story.

Stories likewise give an enthusiastic connection the people and they recollect it until the end of time. At the point when real life stories are expressed to employees they concentrate more on workplace safety.

\subsection{Promoting Safety committee get feedback from employee}

Effective safety committees of executors are various and ought to incorporate workers from all depatment: fabricating, transport, maintenance, front office staff, and so on. They additionally get training on an assortment of safety subjects to make them more mindful of and eliminate workplace safety hazards. Safety managers regularly administer this commitee of executors, however are available in a advising or supporting role only.

A health and safety committee of trustees can be a vital method to enhance conditions at work. The committee gives a group to employees and management to cooperate to take care of health and safety issues. A viable commitee can help prevent injury and illness at work and increment awareness with health and safety issues among laborers, manager and supervisors and create procedures to make the workplace can be safe and healthy.

\subsection{Safety exhibition}

Worker knows hazard in the company, This is another best way to improve worker awareness by providing Safety exhibition. Safety exhibition provided by company what are all the hazard having the company make proto type equipment with safe working procedure. It help to worker recognize hazard in company by seeing proto type equipment.

It can be installed near company entrance because any visitor, contract worker, management staff comes in company after completing safety induction training will be directed to go through the prototype models to understand the safety features better.

\subsection{Safety meeting}

Safety officer has to conduct meeting on work site in every shift before work has to begin, safety officer interact people talk about previous accident happen in company and how to eliminate this kind of hazard because everyone know about hazard happen in company. This is the way to alert worker and doing work carefully without accident.

Monthly once safety officer conduct meeting by calling all worker and management staff, the meeting duration does not exceeding 15 minute. This meeting important to talk about brief about safety and end of the meeting read safety pledge. The safety pledge should have the local language. 


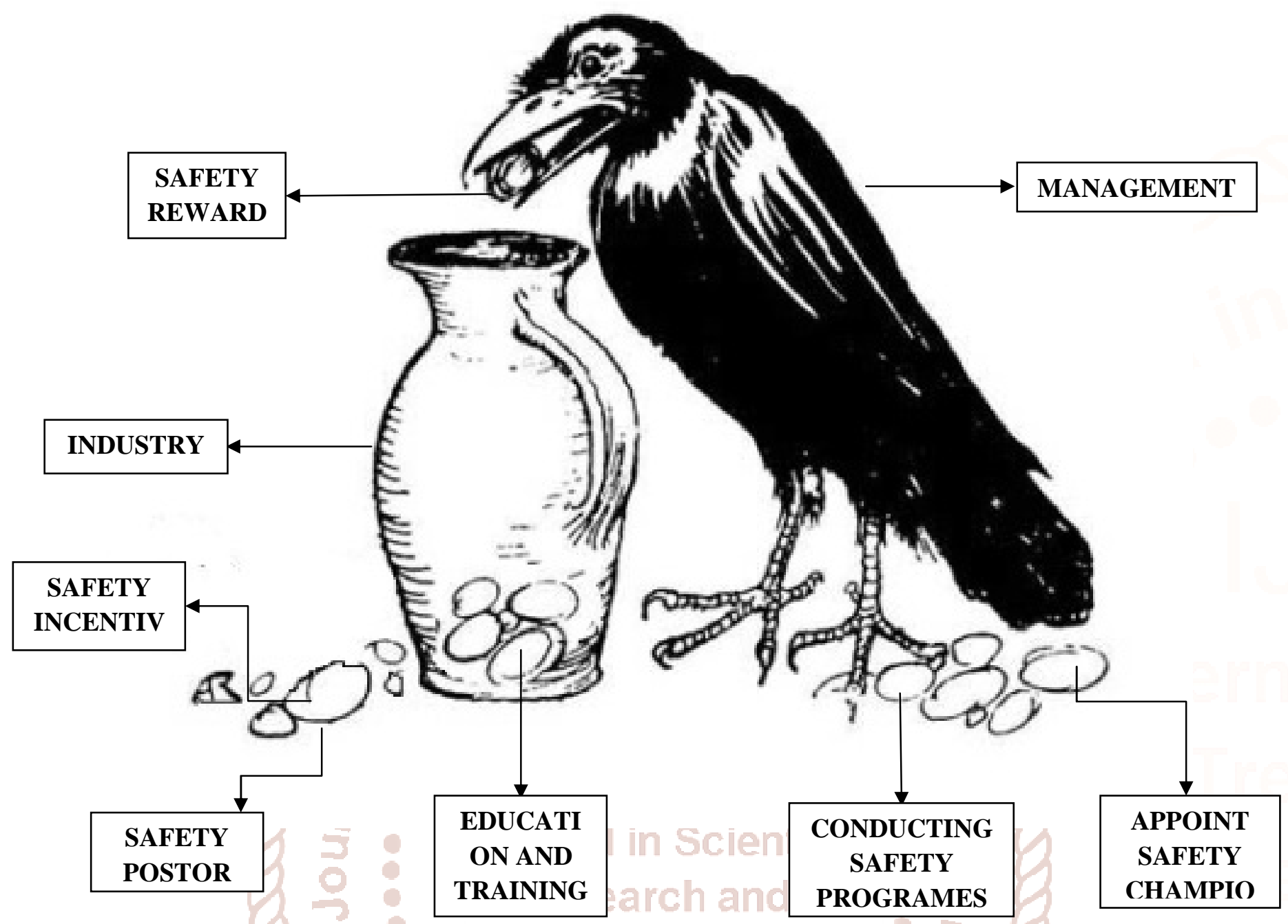

This picture represent, we already heard about this story, the story was the crow returned from a very long trip and was really thirsty. He searched allaround for drinking water; He saw some drinking water inside the pot. But, the crow's beak could not reach the water, as the water level was very low. The crow thinks for a while and anplan arose to him. He thought, 'If I throw stone in the pot, the level of the water would go up. Then, crow will be able of drink the water easily'. Same as assume crow as management, pot as industry, stone as awareness. So Improve worker safety knowledge we have to put awareness on the industry, automatically knowledge of worker safety as grows up easily, significantly reduce human injury as well as increase work place safety.

\section{RESULT AND DISCUSSION}

The research has done in one of the best edible oil manufacturer in India. After implementing all the safety measures within the specific period of time, the response was phenomenal from the workers for the following measures that we implemented:
The Safety induction training

Reward employees for safe behavior

Employee safety incentive plan

$>$ Emotional stories (Because of emotional appeal and hands on training possible in these procedures.)

And following measures yielded good response:

Conducting safety programs with safety related term

$>$ Live demonstrations

$>$ Toolbox training

$>$ Promoting Safety committee

$>$ Implementing Safety Champion

Timely posting of safety information and Establishing safety signs are not having an expecting response from the workers of the company.

\section{Conclusion}

The review of paper done so far has yielded the following points Train-the-Trainer Programs, passive and active training, safety communication, Surprising 
Incentive. The safety culture will be more effective if the methodologies mention in this paper will put into practice such as induction training, education and training, safety related programe, safety reward, Appoint Safety Champion, Employ safety incentive plan, Live demonstrations, Safety committee. The methods mention here are specific to improve safety culture. According to factory act 7A said the provision of such information, instruction, training and supervision as are necessary to ensure the health and safety of all workers at work, it is mandatory to have such training programe focusing on core aspect of safety. Therefore by following above mention methods safety can be improve significantly in any industry

\section{Reference}

1. Talapatra, Subrata, and MdHabibur Rahman. "Safety Awareness and Worker's Health Hazards in the Garments Sector of Bangladesh." European Journal of Advances in Engineering and Technology 3.9 (2016): 44-49.

2. O'Connor, Tom, et al. "Occupational safety and health education and training for underserved populations." New Solutions: A Journal of Environmental and Occupational Health Policy 24.1 (2014): 83-106.

3. Saarela, KaijaLeena. "A poster campaign for improving safety on shipyard scaffolds." Journal of Safety Research 20.4 (1989): 177-185.

4. Slatin, Craig. "Health and safety organizing: OCAW's worker-to-worker health and safety training program." New Solutions: A Journal of Environmental and Occupational Health Policy11.4 (2002): 349-374.

5. Konijn, Astrid M., et al. "The effect of active and passive occupational health and safety (OHS) training on OHS awareness and empowerment to participate in injury prevention among workers in Ontario and British Columbia (Canada)." Safety science 108 (2018): 286-291.

6. Keffane, Salim. "Communication's Role in Safety Management and Performance of the Road Safety Practices." Jordan Journal of Civil Engineering 9.2 (2015).

7. Ghasemi, Fakhradin, et al. "Surprising incentive: An instrument for promoting safety performance of construction employees." Safety and health at work 6.3 (2015): 227-232.

8. Griffin, Mark A., and Andrew Neal. "Perceptions of safety at work: a framework for linking safety climate to safety performance, knowledge, and motivation." Journal of occupational health psychology 5.3 (2000): 347.

9. He, Ahong, SuruiXu, and Gui Fu. "Study on the basic problems of safety culture." Procedia engineering 43 (2012): 245-249.

10. Robson, Lynda S., et al. "A systematic review of the effectiveness of occupational health and safety training." Scandinavian journal of work, environment \& health (2012): 193-208. 